\title{
Evolution properties of online user preference diversity
}

\author{
Qiang Guo ${ }^{\text {a }}$, Lei Ji ${ }^{\text {a }}$, Jian-Guo Liu ${ }^{\mathrm{a}, \mathrm{b}, \mathrm{c}, *}$, Jingti Han ${ }^{\mathrm{b}}$ \\ ${ }^{a}$ Research Center of Complex Systems Science, University of Shanghai for Science and Technology, Shanghai 200093, PR China \\ ${ }^{\mathrm{b}}$ Data Science and Cloud Service Centre, Shanghai University of Finance and Economics, Shanghai 200433, PR China \\ ${ }^{\mathrm{c}}$ Department of Physics, Fribourg University, $\mathrm{CH}-1700$ Fribourg, Switzerland
}

\section{H I G H L I G H T S}

- The evolution pattern of the online user preference is investigated.

- The user rating preference would become diverse and then get centralized finally.

- The correlation between the user ratings and the object qualities keeps increasing.

\section{Introduction}

Understanding the effects of the online collective behavior patterns is important for the computational social science [1], decision-making processes [2-4], and the marketing strategy [5-8]. Recently, the exploration of the online user preferences has become a promising issue for predicting the success of culture products and presenting precise marketing strategy [9-12]. Han et al. [13] investigated the adaptive interests of online user preferences, which is helpful for solving the diversity-accuracy dilemma [14]. Zhang et al. [15] proposed an evolutionary hypergraph model to reconstruct the online user-tag-resource network, showing the user preferences to save resources with tags they are interested in. An evolving network model then was introduced to reproduce the user selection patterns on tags, music and movies [16]. Thanks to the development of web 2.0, online users could not only select, browse, but also review and share what they like. Their preferences and tastes are reflected by their collective rating or selecting behaviors $[17,18]$. Many efforts have been devoted

\footnotetext{
* Corresponding author at: Research Center of Complex Systems Science, University of Shanghai for Science and Technology, Shanghai 200093, PR China. E-mail address: liujg004@ustc.edu.cn (J.-G. Liu).
} 
Table 1

The basic statistical properties of the data sets. $N$ and $M$ is the number of users and objects respectively. The number of links $E$ represents all ratings delivered by users during the time span, denoted by the number of days from the start date to the end date.

\begin{tabular}{|c|c|c|c|c|c|c|}
\hline Data sets & $N$ & $M$ & E & Start date & End date & \#day \\
\hline MovieLens & 71567 & 10681 & 10000054 & 01-09-1995 & 01-05-2009 & 5110 \\
\hline Netflix & 478190 & 17770 & 17197883 & 01-01-2003 & $12-31-2004$ & 731 \\
\hline Amazon & 99621 & 645055 & 2005409 & 05-31-1996 & $09-15-2005$ & 3394 \\
\hline Epinions & 120492 & 755760 & 13668320 & $01-10-2001$ & $05-30-2002$ & 505 \\
\hline
\end{tabular}

to detect the online user preference patterns by analyzing these user generated contents [12,19-21]. Rybski et al. [22,23] analyzed the communication activity and the correlation between the long-term correlation and inter-event clustering. Yang et al. found that there is an anchoring bias effect during the user rating process [24]. Hou et al. [25] investigated the memory effect of the online user rating series [26,27] and found that the Markov model could regenerate online user collective behaviors, which indicates that a user's next action depends only on his/her current behaviors [28-31].

The above works mainly focused on the correlation between the next action and the current one regardless to the user lifespan. How do the online user preferences change throughout the user life span? Whether the Markov model could be commonly used to generate the online behaviors? Inspired by these questions, we investigate the evolution properties of online user preference diversity, where the preference diversity is measured by the variation coefficient. Firstly, we rescale each user life span to an standard time interval (see Methods), which has been used for the domain analysis of various online settings [32-35]. Comparing with the null model, the empirical results indicate that, for movies and reviews, the diversity of user preference increases initially and then decreases to a small value. More importantly, we find that the Markov process could only describe some empirical user rating behaviors when the variation coefficients are stable. Furthermore, we investigate the correlation between user rating value and the object quality. The statistical results suggest that, generally speaking, the correlation between the rating value and the object quality decreases.

\section{Data description}

Four different data sets are introduced in this paper, differing in the subject matter and the span of the time window, as shown in Table 1. These data sets are widely applied to modeling the patterns of online user preferences [24-26]. Each record in these data sets reads the user-object pair, followed by the rating the user gave to the object as well as the corresponding timestamp. The MovieLens ${ }^{1}$ and Netflix ${ }^{2}$ data sets are from websites which accommodate movies of various types for users. Generally, the users would not only watch movies but also give their ratings. The Epinions data set [36] compiles millions of user ratings on expert product reviews (e.g., reviews on electronics, cars and books). The Amazon ${ }^{3}$ data set retains huge volume of user rating records on books. In the MovieLens data set, the user ratings are float number from 0.5 to 5 , which is differentiated into 10 levels with 0.5 score as the difference of adjacent levels. In the other data sets, ratings are integers from 1 to 5 . In this paper, we only take into account the users who rated at least 100 objects with active time span no less than 40 days. Meanwhile, we assume the object quality can be denoted by the ratings average over all of the users.

\section{Methods}

In the above data sets, users can give explicit ratings to the object, expressing to what extent they like or dislike the object. The higher a rating is, the higher the user evaluates the object. Processing the user rating sequence is reasonably regarded as the direct and effective way to reflect the properties of online user preferences. For instance, consecutively delivering higher or lower ratings has been used to identify the anchoring bias and memory effect of user online rating and selecting behaviors [24,25]. In this paper, the evolution properties of online user preference diversity could be measured by the variation coefficient $V$, a widely used distribution-based measure in the field of complex systems [37], defined as the ratio of the standard deviation $\sigma$ to the mean $\mu$. Specifically, we normalize each user's time span into the same time interval [0,1], where 0 and 1 represent the starting and ending time of the online user activities. For a user, say $u$, who delivered $m_{u}$ ratings during his/her time span, the variation coefficient $V$ could be calculated in the following way. Firstly, the normalized time span is divided into $T$ intervals with the same length, which could be denoted as $\left[0, \frac{1}{T}\right),\left[\frac{1}{T}, \frac{2}{T}\right), \ldots,\left[\frac{T-2}{T}, \frac{T-1}{T}\right),\left[\frac{T-1}{T}, 1\right]$, respectively. All the $m_{u}(t)$ ratings before time $t$ consist of the sequence $R S(t)=\left\{r_{1}, r_{2}, \ldots, r_{m_{u}(t)}\right\}$, where $r_{i}$ is the $i$ th rating given by user $u$. Therefore, for each time $t$, the coefficient of variation $V_{u}(t)$ for user $u$ can be expressed as,

$$
V_{u}(t)=\frac{\sigma_{u}(t)}{\mu_{u}(t)}
$$

\footnotetext{
1 http://www.grouplens.org.

2 http://www.netflix.com.

3 http://www.amazon.com.
} 


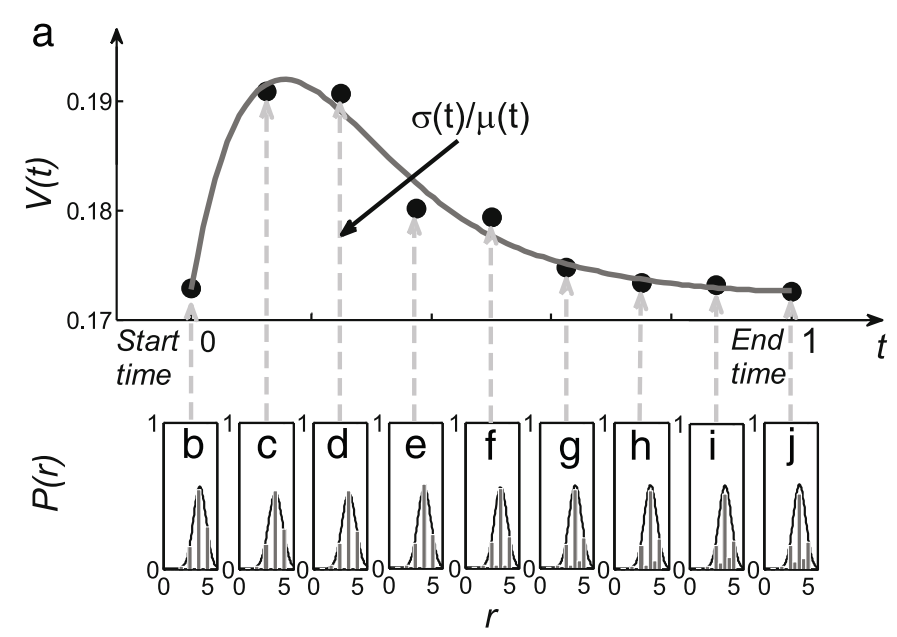

Fig. 1. An illustration of the evolution property of one specific user's preference for the MovieLens data set. The preference diversity is measured by the variation coefficient $V(t)$. The number of movies rated by this user is 295 . And the user stays in the system for 3171 days, which is rescaled to the interval $[0,1]$ and is separated into 9 sub-intervals of same length. Each scatter point in panel (a) indicates the variation coefficient $V(t)$ versus time $t$, which is measured by the ratio of the standard deviation $\sigma(t)$ to the mean value $\mu(t)$, denoted by $\sigma(t) / \mu(t)$. The evolution property of the variation coefficient $V(t)$ can be shown by the gray solid line. The panels (b)-(j) show the distributions $P(r)$ of the user rating sequence for each sub-interval.

where the mean $\mu_{u}(t)$ and the standard deviation $\sigma_{u}(t)$ of the rating sequence $R S(t)$ are read as,

$$
\begin{aligned}
\mu_{u}(t) & =\frac{1}{m_{u}(t)} \sum_{i=1}^{m_{u}(t)} r_{i}, \\
\sigma_{u}(t) & =\sqrt{\frac{1}{m_{u}(t)} \sum_{i=1}^{m_{u}(t)}\left[r_{i}-\mu_{u}(t)\right]^{2} .}
\end{aligned}
$$

Then the higher of the variation coefficient $V_{u}(t)$, the more diverse the user preference would be. When there is not any rating behaviors in one time interval, the $V_{u}(t)$ would be set as zero for user $u$.

For one specific user in the MovieLens data set, the evolution property of the user preference is shown in Fig. 1, from which one could find that, in the early stage, the variation coefficient $V(t)$ increases from 0.1730 to 0.1910 , and then decreases from 0.1910 to 0.1727 . The result indicates that user prefer to give more diverse ratings when they enter the system, and then give stable ratings. From the viewpoint of collective behaviors, the average variation coefficient $\langle V(t)\rangle$ for all users could be defined as,

$$
\langle V(t)\rangle=\frac{1}{N} \sum_{u=1}^{N} V_{u}(t),
$$

where $N$ is the number of users of the data set. Besides, the mean value $\langle\mu(t)\rangle$ is defined as the average rating value over all users, which can be read as,

$$
\langle\mu(t)\rangle=\frac{1}{N} \sum_{u=1}^{N} \frac{\sum_{i=1}^{m_{u}(t)} r_{i}}{m_{u}(t)} .
$$

\section{Evolution properties of online rating preference}

The evolution property of the mean value $\langle\mu(t)\rangle$ is shown in Fig. 2(a)-(d), which is denoted as triangles. For the MovieLensand Amazon data sets, one can find that the mean value $\langle\mu(t)\rangle$ continually decreases as the time $t$ increases, which indicates that the users tend to give smaller rating values as they rated on more objects. Meanwhile, the mean value $\langle\mu(t)\rangle$ of ratings for the Epinions data set monotonously increases with the user comment activities. However, for the Netflix data set, the evolution of the mean value $\langle\mu(t)\rangle$ exhibits another pattern, in which users give lower ratings on movies in their early stage, then give higher scores in the rest of life span.

The dynamics of the mean value $\langle\mu(t)\rangle$ show the evolution property of the rating values. Furthermore, we investigate the variation coefficient to measure the rating diversity of all users (see Methods). Fig. 2(e)-(h) show the evolution processes of the average variation coefficient $\langle V(t)\rangle$ in different systems, from which one could find that, except the Amazon data set, the 

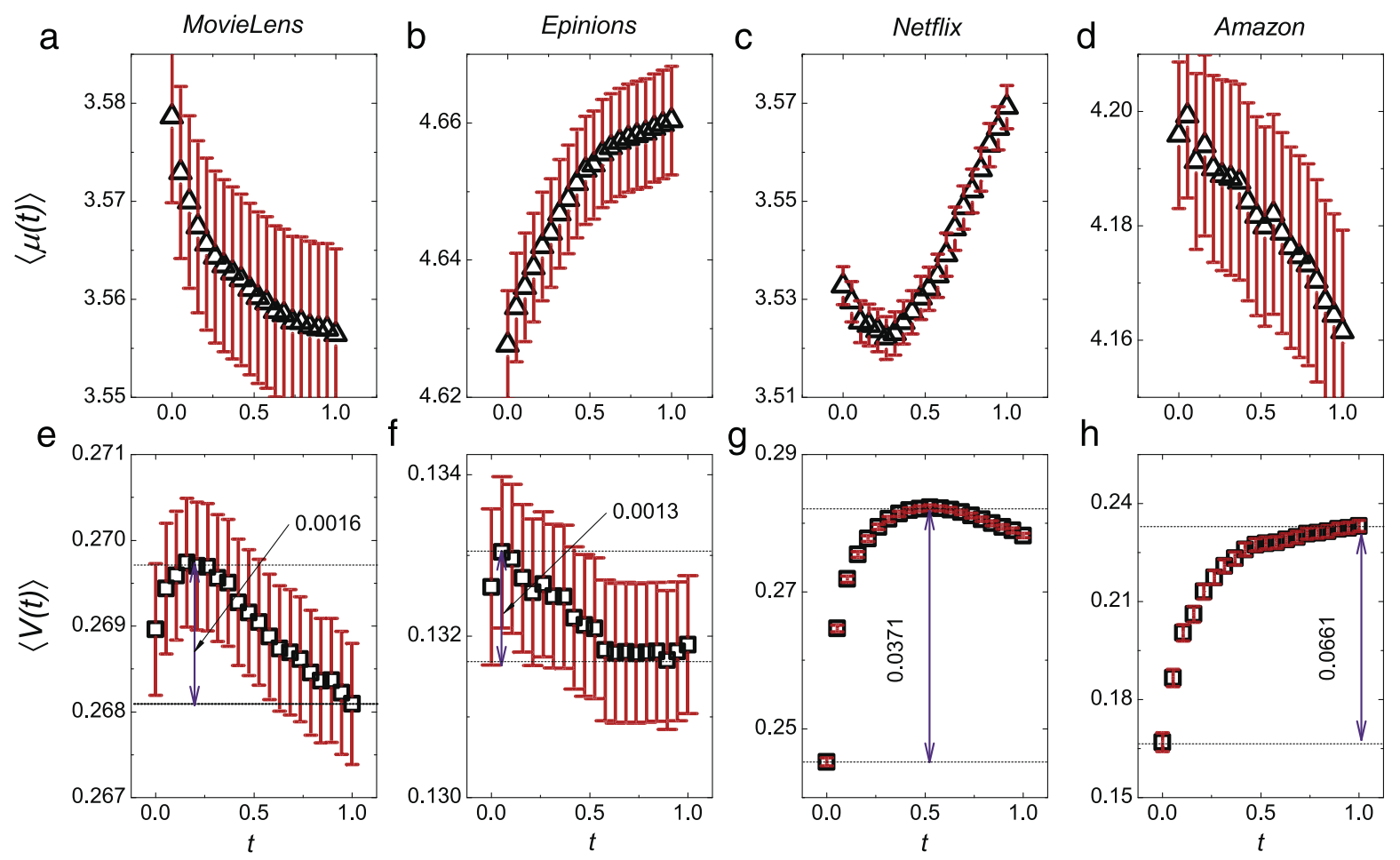

Fig. 2. (Color Online) The evolution processes of the average variation coefficient $\langle V(t)\rangle$ and the mean value $\langle\mu(t)\rangle$ across the user activity time span. The activity time span is mapped into the range $[0,1]$, where 0 and 1 indicate the first and last rating times. From the panels (a)-(d), one could find that, the mean value $\langle\mu(t)\rangle$ for the MovieLens and Amazon data sets decreases continually, which means the users tend to give small values on the objects. On the contrary, for the Epinions data set, the users would gradually give high scores on the others' review. For the Netflix data set, the mean value $\langle\mu(t)\rangle$ decreases at the early stage, then rapidly increases finally. Apparently, there is different dynamics of the user rating preference in different systems. Furthermore, the variation coefficient of the user rating sequence is introduced. Panels $(\mathrm{e})-(\mathrm{h})$ show the dynamics of the average variation coefficient $\langle V(t)\rangle$, from which one find that, except the Amazon data set, the average variation coefficient $\langle V(t)\rangle$ increases firstly and then continually decreases. However, for the Amazon data set, the average variation coefficient $\langle V(t)\rangle$ continually increases. The results suggest that, for movies/reviews, the preference tends to be more and more diverse and then increasingly centralized eventually. The red vertical bar indicates the standard error bar.

average variation coefficient $\langle V(t)\rangle$ increases in the early stage of the time span, then reaches the maximum, and eventually declines. From Fig. $2(\mathrm{e})-(\mathrm{h})$, one could find that, the value ranges of the average variation coefficient $\langle V(t)\rangle$ are 0.0371 and 0.0661 for the Netflix and Amazon data sets, which are at least 22 times of the value ranges for the MovieLens and Epinions data sets. The remarkable difference of the value ranges suggests that the user preference diversity is more stable for the MovieLens and Epinions data sets than the diversity for the Netflix and Amazon data sets. Therefore, for the Netflix and Amazon data sets, the disability of the Markov process to regenerate the properties of online user behaviors could result from both the notable diverse trend of the user's preference and the large value range of the variation coefficient.

The results indicate that, there is no specific preference for movies/reviews when users just enter the system. As the experiences get more extensive they prefer to rate or select the specific movies or reviews they like, thus it appears that their preferences become more and more centralized. For the Amazon data set, nevertheless, the average variation coefficient $\langle V(t)\rangle$ for books tends to increase continually, indicating the user preferences on books are growing diverse. In addition, we present a null model to compare the empirical and reshuffled results, which demonstrates the evolution patterns shown in Fig. 2 are from real online user behaviors instead of the random mechanism. In the null model, each user would randomly deliver integer ratings $(1,2, \ldots, 5)$ on the objects while the links and timestamps for each user-object pair unchanged. Therefore, the empirical dynamics for each user's rating preference are removed. The dynamical patterns of the average coefficient of variation $\langle V(t)\rangle$ for the null model are shown in Fig. 3. It can be seen that, for all four data sets, the average coefficient of variation $\langle V(t)\rangle$ keeps increasing as the time increases. Apparently, for the MovieLens, Epinions, and Netflix data sets, the empirical results of the evolution processes of the average coefficient of variation $\langle V(t)\rangle$ is remarkably different with the results obtained by the null model, which suggests that the centralized trend of rating preference for movies and reviews cannot be regenerated by the user's random rating behaviors. On the contrary, in the Amazon data set, the difference of the evolution trends between real data and the null model is not significant, which indicates that the rating behaviors for books are more random than that for movies or reviews.

For user with different degrees, would the evolution patterns of the user rating preference still behave uniformly as the ones in the main text? Hence, for each data set, we group the users according to their degrees (the number of ratings they delivered during the activity span), and implement the experiments for the dynamics of the average coefficient of variation $\langle V(t)\rangle$ for different groups. 

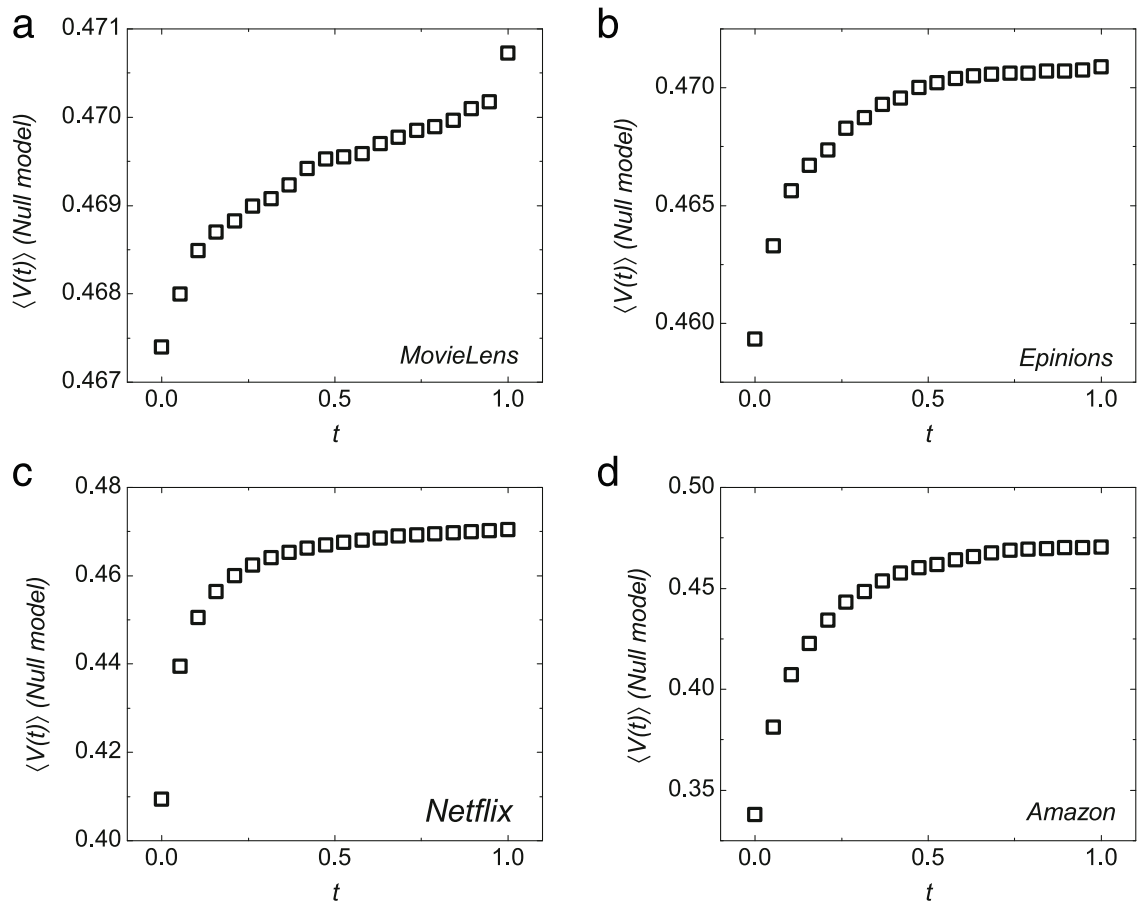

Fig. 3. Evolution dynamics of the coefficient of variation $\langle V(t)\rangle$ for the null model. A null model is proposed to comparing with the empirical results. The null model is constructed by uniformly randomizing the user ratings from the integral number range $[1,5]$. Meanwhile, the links and timestamps for each user-object pair stay the same as the empirical ones. The statistical results of the evolution dynamics of the coefficient of variation $\langle V(t)\rangle$ are shown in panels (a)-(d), from which one could find that, the coefficient of variation $\langle V(t)\rangle$ monotonously increases with the increment of time $t$ for each data set. Therefore, for the MovieLens, Epinions, and Netflix data sets, the evolution patterns of the online user preference cannot be regenerated by the users' randomized behaviors. Whereas, for the Amazon data sets, the process of increasing diversification of the user online preference appears more random than regular.

For each data set, users are collected into six groups depending on their degree $k$. Specifically, considering the users in a certain group $g(g=1,2,3,4,5)$, the degree range of the users in this group is denoted as $[k(g), k(g) \times d]$, where the common ratio $d=\frac{k(g+1)-1}{k(g)}$ with $k(1)=100$, and the degree range for the last group could be $\left[k(5)+1, k_{\max }\right]$. Then we calculate the average coefficient of variation $\langle V(t)\rangle$ for different group during each time interval. For an arbitrary time interval $\left[t-\frac{1}{T}, t\right)$, (where the time $t \in\left\{\frac{1}{T}, \frac{2}{T}, \ldots, \frac{T-1}{T}, 1\right\}$ ), the average coefficient of variation $\langle V(t)\rangle_{g}$ for group $g$ can be read as,

$$
\langle V(t)\rangle_{g}=\frac{1}{N_{g}} \sum_{u \in \Omega_{g}} V_{u}(t),
$$

where $N_{g}$ is the number of users in group $g, \Omega_{g}$ is the set of users in group $g$, and $V_{u}(t)$ denotes the average coefficient of variation for an arbitrary user, say $u$, during the time interval. Therefore, at the time $t$, the higher the value of the average coefficient of variation $\langle V(t)\rangle_{g}$ is, for the Group $g$ the more diverse the users rating preferences are. Moreover, to compared with the empirical results, we also implement the experiments for the null model to the corresponding groups. For different data sets, we discuss the effect of the user degree on the evolution patterns of the online user preference, the detailed results are reported as follows.

\subsection{Results for the MovieLens data set}

In the MovieLens data set, the common ratio $d=1.53$ so that the degree ranges from the least to the largest for different groups are [100, 153], [154, 236], [237, 363], [364, 558], [559, 857] and [858, 7359] respectively. The effect of the user degree on the evolution dynamics of user preference is shown in Fig. 4(a). The users with large-degree are likely to have higher values of the average coefficient of variation $\langle V(t)\rangle$. Compared with the small-degree users, the preference for the large-degree users are more diverse, which indicates that, the users with more extended rating and selecting experience on movies would intend to browse or review the movies of various kinds. As the time $t$ increases, for users in group 1 and group 3 , the average coefficient of variation $\langle V(t)\rangle$ exhibit slightly decreasing tendency over the activity span. For users in group 2 and group 4, the coefficient of variation $\langle V(t)\rangle$ presents little fluctuation. While for the large-degree users in group 5 and group 6, the coefficient of variation $\langle V(t)\rangle$ tends to increase to the maximum value first, and then decrease to a stale value. 

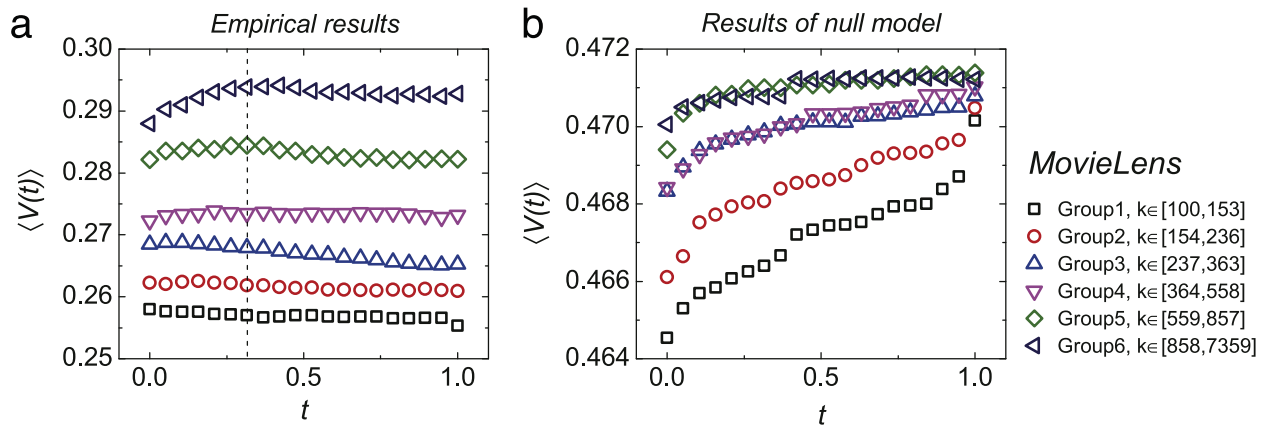

Fig. 4. (Color online)The evolution dynamics of average coefficient of variation $\langle V(t)\rangle$ for different user groups in the MovieLens data set. Users are grouped into 6 groups by their degrees that range for different groups in [100, 153], [154, 236], [237, 363], [364, 558], [559, 857] and [858, 7359] respectively. The panel (a) shows the empirical results for the evolution dynamics of the average coefficient of variation $\langle V(t)\rangle$. For users in group 1 and group 3 , the average coefficient of variation $\langle V(t)\rangle$ decreases monotonously and slightly. For users in group 2 and group 4 , the average coefficient of variation $\langle V(t)\rangle$ fluctuates slightly around the first value. Whereas, for the users in group 5 and group 6 , the average coefficient of variation $\langle V(t)\rangle$ start to increase at first till the maximum value, and then decreases eventually. The panel (b) exhibits the evolution dynamics of the average coefficient of variation $\langle V(t)\rangle$ for null model. As one could find, the average coefficient of variation $\langle V(t)\rangle$ increases for all the user groups throughout the whole time span. The comparison between the empirical results and the ones for null model indicates that, in real rating process the centralized trends of the user preference cannot be regenerated by the users' randomized behaviors, especially for the large-degree users. Moreover, the average coefficient of variation $\langle V(t)\rangle$ lies in range [0.2554, 0.2941$]$ in the empirical case, while it locates in range $[0.4645,0.4714]$ for the null model, which indicates that the real user preference is much more centralized than that of the null model.

Thus, for users with little experiences in rating and selecting movies, the diversity of their preference are likely to keep on a certain level. Whereas, with the extended experience of users' online rating and selecting activities, their preference would eventually present more and more centralized trend.

For the null model, the Fig. 4(b) displays the effect of the user degree on the evolution characteristics of online user preference. It could be seen that, for each group of users with different user degree, the average coefficient of variation $\langle V(t)\rangle$ would be monotonously increases with time $t$. Therefore, if users randomly deliver the ratings on movies without their real and specific preference, the tastes would be increasingly diverse. Moreover, in the empirical case, the average coefficient of variation $\langle V(t)\rangle$ locates in range [0.2554, 0.2941] for different users, while in the null model, it ranges from the minimum value 0.4645 to the maximum 0.4714 . Apparently, in the empirical condition, the user rating is much more centralized than that of the null model, which indicates the remarkable preference in the user rating behaviors. Therefore, for the MovieLens data set, comparing the results of null model with the empirical ones, we could conclude that, the centralized trend of the user preference are significant, especially for the large-degree users. In fact, the user preference is much more diverse in null model than that in empirical conditions.

\subsection{Results for the Epinions data set}

For the Epinions data set, the degrees for different groups belong to the ranges [100, 209], [210, 438], [439, 918], [919, 1923], [1924, 4027] and [4028, 162 169] respectively, in that the common ratio $d$ equals 2.09. For different groups, the evolution dynamics of the average coefficient of variation $\langle V(t)\rangle$ are shown in Fig. 5 for both the empirical data and the null model. Note that in the empirical results, there is no significant tendency that the large-degree users have more diverse preference than the small-degree users or vice versa. For instance, the value of the average coefficient of variation $\langle V(t)\rangle$ for the users in group 3 is lower than that for the users in group 1 while it can be much higher than the values for users in group 2. Furthermore, as we could find, for users in group 1, group 2, and group 4, the average coefficient of variation $\langle V(t)\rangle$ increases in the early stage of user activity time span, while it would continuously decreases till the end. For users in group 3 and group 5, the values of the average coefficient of variation $\langle V(t)\rangle$ monotonously decreases. However, the result for group 6 shows remarkable different evolution pattern. The average coefficient of variation $\langle V(t)\rangle$ declines to the minimum value from the start of the activity time span, and then increases in the rest of time. Therefore, the empirical results implies that, for most users their preferences present more and more centralized trend. For users with degree $k>4027$, however, the dynamical evolution process of the preference behave in a pattern that is opposite to the small-degree users.

Similarly, we implement the analysis for the null model. The results are shown in Fig. 5(b), in which the average coefficient of variation $\langle V(t)\rangle$ rapidly increases from the start of the user activity time span and slightly increases to the end. Apparently, the remarkably different evolution patterns between the empirical results and the results of null model demonstrate the significance of our analysis in the main text. In addition, the average coefficient of variation $\langle V(t)\rangle$ for the group 6 increases eventually, which could explain the little fluctuation of the average coefficient of variation $\langle V(t)\rangle$ after $t=0.5890$ in Fig. $2(\mathrm{f})$ in the main text. Besides, we can also find that, the value range of the average coefficient of variation $\langle V(t)\rangle$ is $[0.1249,0.1387]$ for different groups. However, the minimum value of the $\langle V(t)\rangle$ for the null model is 0.4530 , which is over three times than the maximum 0.1387 of the empirical one. Therefore, in the Epinions data set, the user rating preference is also significantly centralized than the much diverse case of null model. 

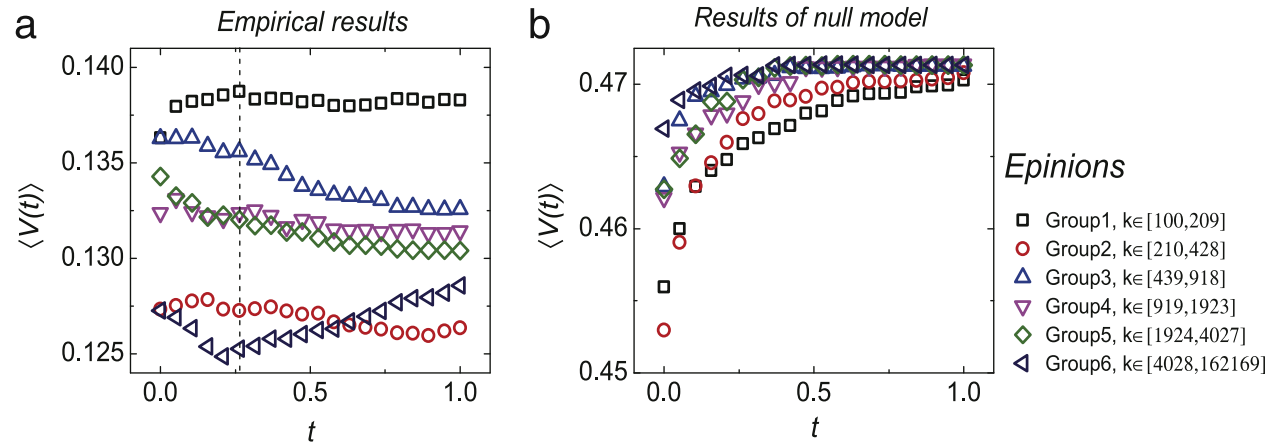

Fig. 5. (Color Online) The evolution dynamics of the average coefficient of variation $\langle V(t)\rangle$ for different users in the Epinions data set. The common ratio $d$ equals 2.09 for the Epinions data set, so that the users are collected into 6 groups by their degrees ranging in [100, 209], [210, 438], [439, 918], [919, 1923], $[1924,4027]$ and $[4028,162169]$ respectively. The panel (a) shows the empirical results for the dynamics of the average coefficient of variation $\langle V(t)\rangle$. Specifically, for users in the group 1, group 2, and group 4, the average coefficient of variation $\langle V(t)\rangle$ tends to increase from the start till the maximum value and then decreases to the end. For users in the group 3 and group 5 , the value of the average coefficient of variation $\langle V(t)\rangle$ decreases monotonously. Whereas, for the users in group 6, the average coefficient of variation $\langle V(t)\rangle$ decreases first till the minimum value and then increases to the end. Panel (b) displays the corresponding results for the null model, in which the average coefficient of variation $\langle V(t)\rangle$ continuously increases. Comparing with the results for the null model, the centralized evolution patterns for users with degree $k<4028$ are significant. However, for users with degree $k \geq 4028$, the evolution process could be in the opposite way. Besides, in the null model, the value of the average coefficient of variation $\langle V(t)\rangle$ is at least over 0.45 , which is three times more than the empirical one.
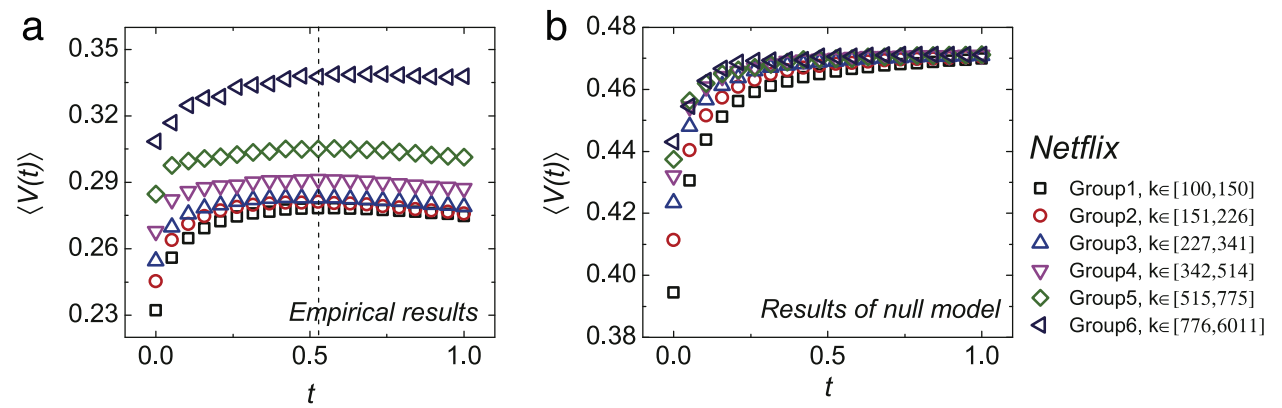

Fig. 6. (Color Online) The evolution dynamics of the average coefficient of variation $\langle V(t)\rangle$ for different users in Netflix data set. For the Netflix data set, the users are collected by the degree ranging in [100, 150], [151, 226], [227, 341], [342, 514], [515, 775] and [776, 6011] into 6 groups. The empirical evolution dynamics of the average coefficient of variation $\langle V(t)\rangle$ is shown in panel (a), in which except the users in group 6 , the value of the average coefficient of variation $\langle V(t)\rangle$ increases till the maximum value and then decreases to the end. For group 6 , the average coefficient of variation $\langle V(t)\rangle$ changes with little fluctuations in the end of the activity time span. In panel (b), however, the average coefficient of variation $\langle V(t)\rangle$ tends to monotonously increases over the whole activity time span. Meanwhile, compared the results in the empirical data with that of null model, one could find that, the maximum of the average coefficient of variation $\langle V(t)\rangle$ in the empirical case is less than the minimum one in the null model, which indicates that in the real rating process the user rating preference is much more remarkable.

\subsection{Results for the Netflix data set}

Similar with the MovieLens data set, each record in the Netflix data set reflect the user's preference on a specific movie. The common ratio $d=1.50$, then the degree for different groups locates in ranges [100, 150], [151, 226], [227, 341], [342, $514],[515,775]$ and $[776,6011]$ respectively. We show the evolution patterns of the average coefficient of variation $\langle V(t)\rangle$ for different groups, the results are presented in Fig. 6(a), from which one could find that, for a fixed time, the values of the average coefficient of variation $\langle V(t)\rangle$ for large-degree users are higher than that for the users of small-degrees. This is consistent with the results for the MovieLens data set. In addition, except the result for group 6, the average coefficient of variation $\langle V(t)\rangle$ keeps increasing from the start of the users' activity time span, and then slightly decreases to a stale value. For the users in group 6 , the values of the average coefficient of variation $\langle V(t)\rangle$ keeps the maximum value after time $t>0.5263$. Thus, the evolution dynamics for different groups almost remain the general pattern similar with that in the main text.

We show the results of null model for the Netflix data set in Fig. 6(b), in which for different users, the average coefficient of variation $\langle V(t)\rangle$ keeps increasing over the whole activity time span. That is, in the Netflix data set, even for the users with different degrees, the empirical evolution patterns of online user preference cannot be regenerated by the users' randomized behaviors. Furthermore, compared the results of null model with the empirical one, we can find that, the maximum value of the average coefficient of variation $\langle V(t)\rangle$ is no more than 0.35 for the empirical data, however, the minimum value for the null model is no less than 0.38 . Thus, for the Netflix data set, the preference of user rating behaviors is reexamined to be much more significant than that of null model. 

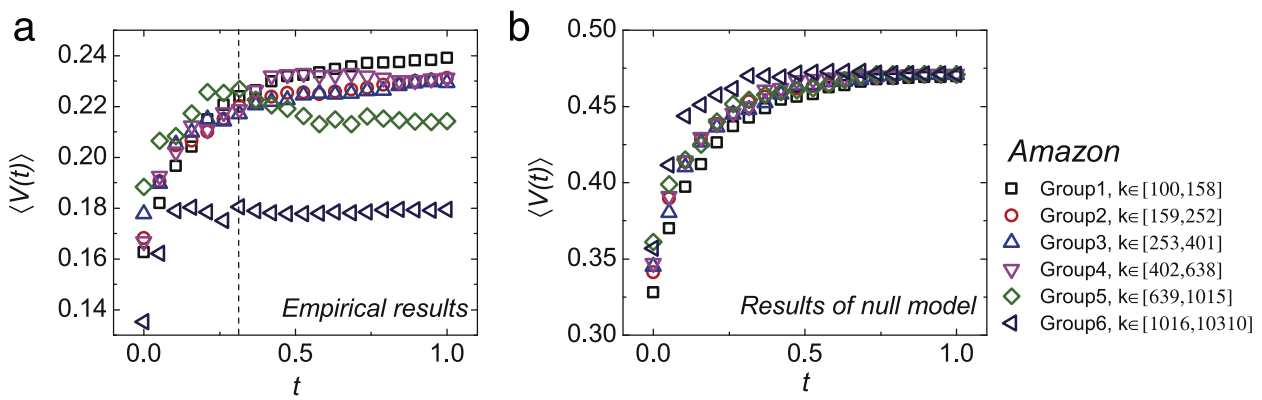

Fig. 7. (Color Online) The evolution dynamics of the average coefficient of variation $\langle V(t)\rangle$ for different users in Amazon data set. The users are collected into 6 groups with the degree locating in ranges [100, 158], [159, 252], [253, 401], [402, 638], [639, 1015], and [1016, 10310] respectively. The panel (a) shows the empirical results for the evolution dynamics of the average coefficient of variation $\langle V(t)\rangle$ for different groups. For the first four groups, the average coefficient of variation $\langle V(t)\rangle$ increases with time over the whole time span. For the users in group 5 , the average coefficient of variation also increases to the maximum value from the start of the activity time span, while decreases during the rest of time. For the users in group 6 , the average coefficient of variation $\langle V(t)\rangle$ first rapidly increases to the maximum value, and then evolves with little fluctuation to the end. The panel (b) shows the corresponding results for the null model. As we could find that, except group 5 , the evolution dynamics for most users are similar with the pattern in the null model. Besides, the minimum value of the average coefficient of variation $\langle V(t)\rangle$ in null model is 0.3279 for different groups, which is significantly larger than the maximum one in the empirical data. The result indicate that, the preference in the real rating process is much significant than that for the randomized case.

\subsection{Results for the Amazon data set}

For the Amazon data set, in the main text we show that the users' preferences are more and more diverse since the average coefficient of variation $\langle v(t)\rangle$ increases over the whole activity time span. In details, we analyze the evolution dynamics of the average coefficient of variation $\langle V(t)\rangle$ for different users. The users are also divided into 6 groups by their degrees, in which the degree ranges in [100, 158], [159, 252], [253, 401], [402, 638], [639, 1015], and [1016, 10 310] respectively. For the first four groups, the average coefficient of variation $\langle V(t)\rangle$ monotonously increases, this type of evolution process is consistent with the dynamical patterns reported in the main text. For users in group 5 , the average coefficient of variation $\langle V(t)\rangle$ increases to the maximum value from the start of the activity time span, and then decreases, which is similar with the evolution patterns of the counterparts in the MovieLens and Epinions data sets. For the users in group 6, the average coefficient of variation $\langle V(t)\rangle$ rapidly increases to the maximum value, and then keep this value to the end. The statistical results indicates that, for users with certain degrees (group 5), their preference could also be more and more centralized as the time increases, while for most users, their preference appears increasingly diverse. (See Fig. 7.)

The experiments for the null model also show that, the average coefficient of variation $\langle V(t)\rangle$ tends to monotonously increase for different groups, and the values (the minimum for null model is 0.3279) are always much higher than that of the empirical ones (the maximum for the empirical data is 0.2391 ). Therefore, the evolution dynamics of the online user preference are far from the ones generated by the randomized behaviors while the user rating preference is far more obvious than the diverse one of null model.

\section{Modeling user rating behaviors via the Markov process}

The Markov model has been widely used to model online user behaviors, while Meiss et al. argued that online user behaviors could not be explained by the Markov model [33]. However, quite a lot of recent advances demonstrated that the user rating and selecting behaviors present considerable correlations with the historical ones (e.g., Anchoring bias effect and Memory effect of online user rating behaviors), indicating the Markovian property implicit in the user rating sequence $[24,25,29,30]$. Therefore, in this paper, we try to regenerate the dynamical rating process for each data set with a Markov model, where the relationship between the user's two consecutive actions are calculated based on the transition matrix, in which the distribution of the transition probability is captured by the Gaussian distribution [25,26]. Suppose the user $u$ delivered $m_{u}^{\prime}(t)$ ratings during a given time interval $\left[t-\frac{1}{T}, t\right)\left(t=\frac{1}{T}, \frac{2}{T}, \ldots, \frac{T-1}{T}\right)$ or $\left[\frac{T-1}{T}, 1\right]$. Then the next rating $r_{i+1}(t)$ given by user $u$ could be given as,

$$
f\left(r_{i+1}(t)\right)=\frac{1}{\alpha\langle\sigma(t)\rangle \sqrt{2 \pi}} \exp \left\{-\frac{\left[r_{i+1}(t)-r_{i}(t)\right]^{2}}{2[\alpha\langle\sigma(t)\rangle]^{2}}\right\},
$$

where $\langle\sigma(t)\rangle=\frac{1}{N} \sum_{u=1}^{N} \sigma_{u}(t)$ is the averaged standard deviation of the ratings over all users until time $t, \alpha$ is an adjustable parameter. Specially, we set all ratings delivered by the user in the first time interval as the basis and the simulations start at the second one. In each time interval, the value of the standard deviation $\langle\sigma(t)\rangle$ is given by the empirical results.

For the simulated ratings of each data set, we calculate the dynamics of the mean value $\langle\mu(t)\rangle$ with different values of $\alpha$. As shown in Fig. 8, the empirical dynamics of the mean value $\langle\mu(t)\rangle$ could be well fitted by the simulated data in the MovieLens and Epinions data sets, with the $\alpha=1.00$ and 1.06 respectively. For the Netflix and Amazon data sets, we could not find a parameter $\alpha$ to regenerate the empirical results. In order to intuitively compare the simulation results, we implement the simulations with the parameter $\alpha$ lying in the range $[0.2,1.6]$ and the value resolution is 0.2 for all the data sets. 

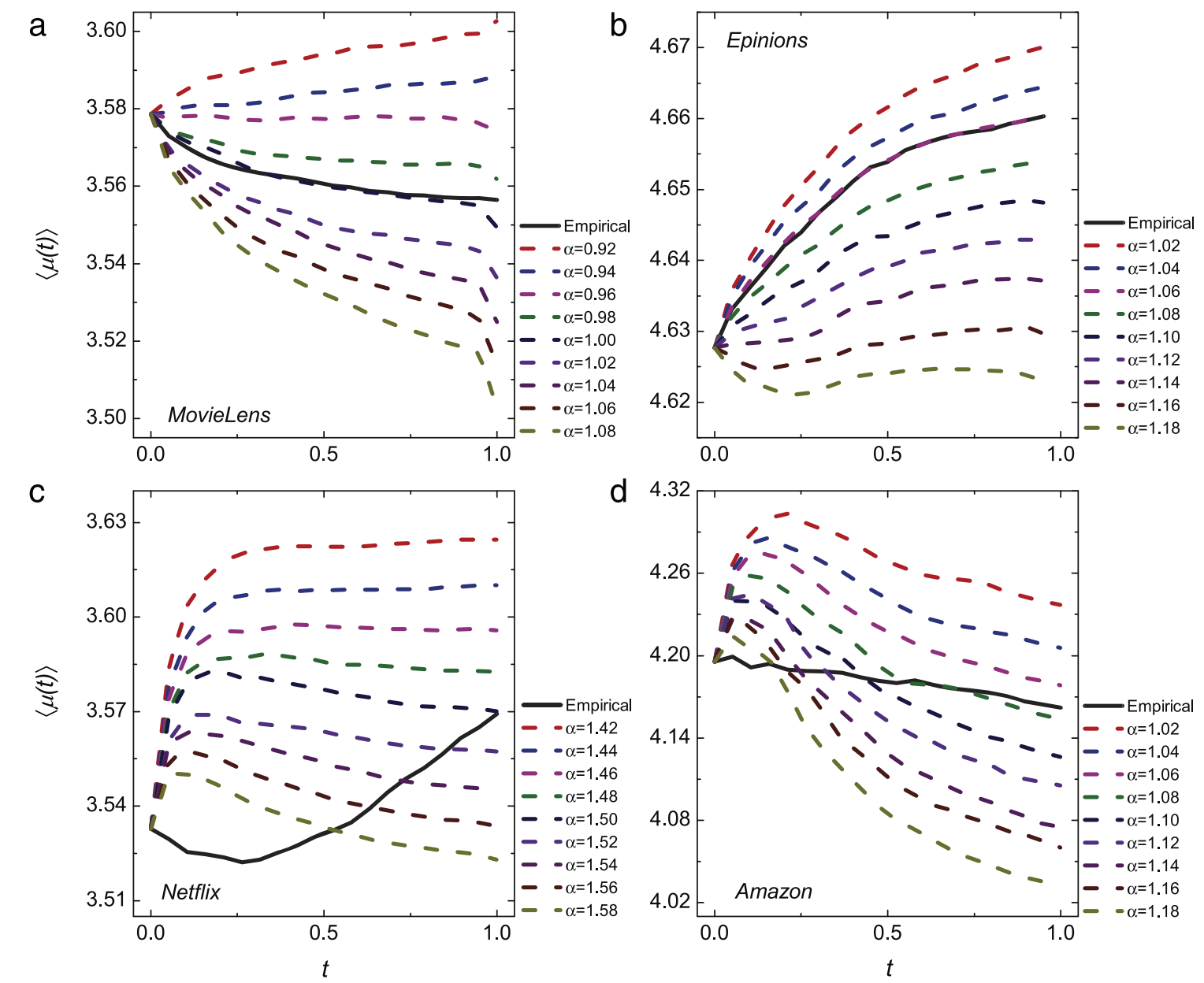

Fig. 8. (Color Online) Regenerating the dynamics of the mean value $\langle\mu(t)\rangle$ via the Markov process with different parameters $\alpha$. The Markov process is widely used to model the correlation between online users' consecutive behaviors $[24,25,29,30]$. The panels (a)-(b) shows that, for the MovieLens and Epinions data sets the dynamics of the mean value can be well regenerated by the Markov process with $\alpha=1.00$ and 1.06 respectively. However, the panels (c)-(d) present that the Markov process could not regenerate the online user behaviors even with various parameters, suggesting that the ability of the Markov process in describing the user behavior patterns could be affected by the evolution characteristics of online user preference.

The results of the simulations are shown in Fig. 9, from which one could find that, the empirical dynamics of the mean value $\langle\mu(t)\rangle$ can be well fitted by the Markov process with the parameter $\alpha=1.0$ and 1.06, respectively for the MovieLens and Epinions data sets. Whereas, for the Netflix and Epinions data sets, the Markov process between the user consecutive actions cannot capture the nature of the user rating dynamics, since the lack of the appropriate value for the parameter $\alpha$. The results are consistent with the ones in the main text. Therefore, the simulation results are robust to the value range of the parameter $\alpha$. In fact, as we shown in Fig. 2, the value of the average coefficient of variation $\langle V(t)\rangle$ for the Netflix and Amazon data sets locates in a much broader value ranges and is higher than the ones for the MovieLens and the Epinions data sets, which may be the reason accounting for the different regenerating results. Another reason for the distinct abilities of the Markov process to reproduced the dynamics of the user behaviors could be attributed to different evolution process of the user rating preference, in which the preference tends to be more and more centralized in the MovieLens and Epinions data sets while it would be increasingly diverse for a much longer periods or over the activity time span in the Netflix and Amazon data sets.

Moreover, in the real rating or selecting process users are usually exposed to a time-varying systems, where the average rating of objects they perceived is also time-dependent. Therefore, the information they could consider when they rated an object is the average rating delivered by other users before. Hence, we explore the correlations between the user rating and the object quality in two steps as follows.

\section{Correlations between the user rating and the object quality}

Firstly, we examine the correlations between the user rating and the object quality, which is defined as the average value over all ratings that the object received. For a specific user $u$ who delivered $m_{u}$ ratings, the rating sequence and the 

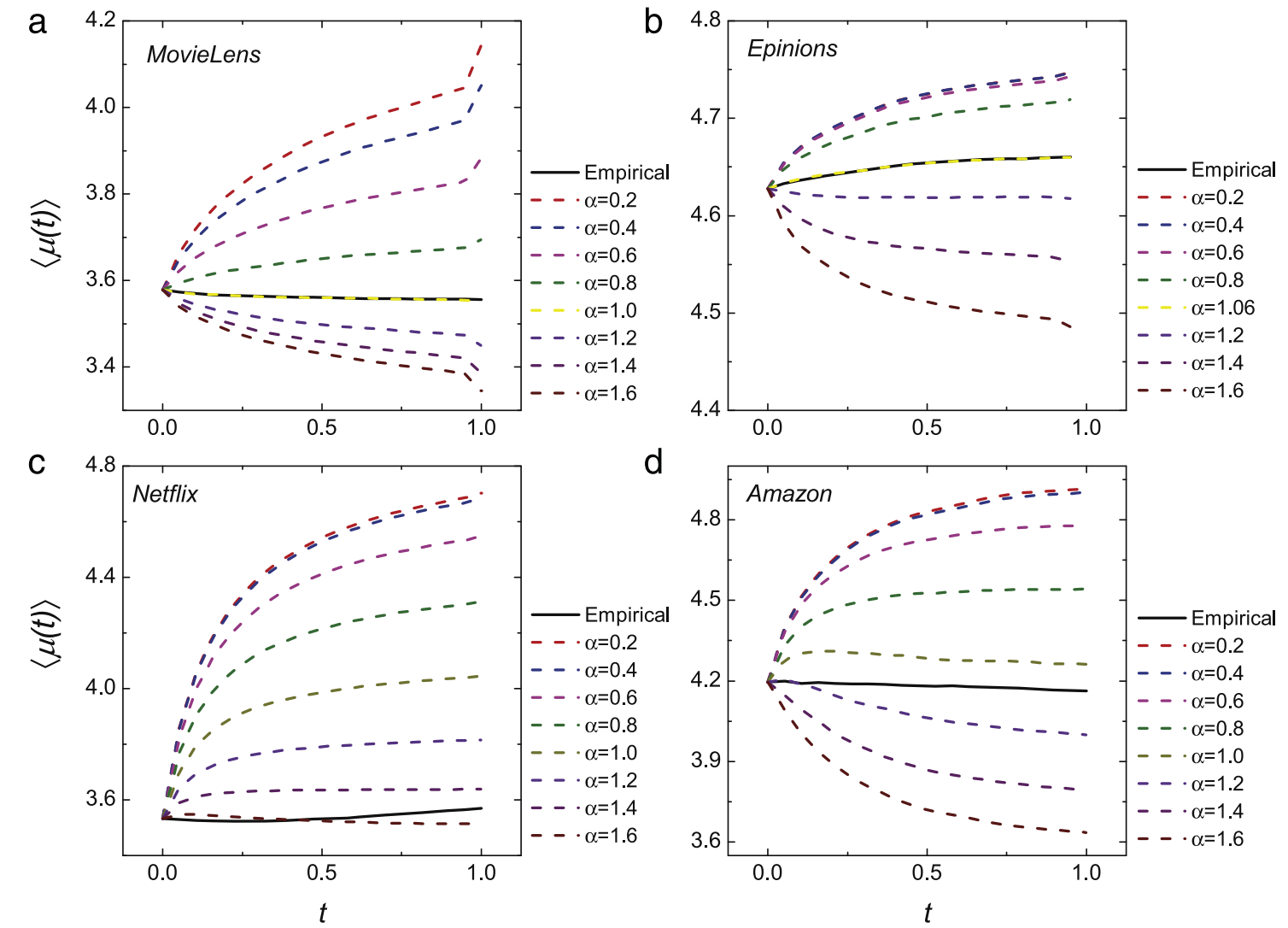

Fig. 9. Simulating the dynamics of the mean value $\langle\mu(t)\rangle$ with the Markov process at the parameter $\alpha$ belonging to a more wide range. For different data sets, the parameter $\alpha$ are set into the same and more broad range [0.2, 1.6], which contains the ones in the main text. Therefore, the simulation results could be equally and intuitively compared over different systems. The results shown in panels (a)-(b) indicate that, for the MovieLens and Epinions data sets, the evolution dynamics of the mean value $\langle\mu(t)\rangle$ can be well described with the Markov type of dynamics with parameter $\alpha=1.0$ and 1.06 . However, panels (c)-(d) show that, for the Netflix and Amazon data sets, the Markov type of dynamics solely cannot reflect the mechanisms of the online user collective behaviors since the lack of an appropriate value of the parameter $\alpha$. The above results are consistent with that in the main text, in which the parameter $\alpha$ is in a more fine-grained resolution and more narrow value range.

corresponding object quality sequence can be denoted as two vectors, $R_{u}=\left\{r_{1}, r_{2}, \ldots, r_{m_{u}}\right\}$ and $Q_{u}=\left\{q_{1}, q_{2}, \ldots, q_{m_{u}}\right\}$ respectively. To evaluate the similarity between the ratings and the qualities, we calculate the Pearson correlation coefficient between the vectors $R_{u}$ and $Q_{u}$. Thus the rating similarity $\theta_{u}$ for user $u$ is defined as,

$$
\theta_{u}=\frac{1}{m_{u}} \sum_{i=1}^{m_{u}} \frac{\left(r_{i}-\bar{r}_{u}\right)\left(q_{i}-\bar{q}_{u}\right)}{\sigma_{r_{u}} \sigma_{q_{u}}},
$$

where $\bar{r}_{u}=\frac{1}{m_{u}} \sum_{i=1}^{m_{u}} r_{i}, \bar{q}_{u}=\frac{1}{m_{u}} \sum_{i=1}^{m_{u}} q_{i}, \sigma_{r_{u}}=\sqrt{\frac{1}{m_{u}} \sum_{i=1}^{m_{u}}\left(r_{i}-\bar{r}_{u}\right)^{2}}$ and $\sigma_{q_{u}}=\sqrt{\frac{1}{m_{u}} \sum_{i=1}^{m_{u}}\left(q_{i}-\bar{q}_{u}\right)^{2}}$. The rating similarity $\theta_{u}$ ranges from -1 to 1 , in which -1 means that the ratings delivered by user $u$ is totally opposite to the object quality, while 1 corresponding to the consistent case. Besides, if the rating similarity $\theta_{u}$ equals 0 , there would be no correlation between user's rating behaviors and the object qualities.

For all four data sets, we calculate the rating similarity $\theta$ of each user. By displaying the probability distribution $p(\theta)$ of the rating similarity $\theta$, Fig. 10 shows the positive correlation between the user ratings and the object qualities. For the MovieLens and Netflix data sets, the modes of the rating similarity $\mu(\theta)$ are 0.52 and 0.46 as well as the mean values are equal to 0.4754 and 0.4373 respectively. Obviously, the results suggest that, a high rating score on movies has a higher possibility of being given to an object of high quality and vice versa, which is consistent with the previous studies [25]. This phenomenon is more evident for rating reviews and books in the Epinions and Amazon data sets as the modes are 0.65 and 0.84 respectively and both of the mean values are beyond 0.5 .

For each user, we separated each user's rating sequence into $T$ intervals by uniformly dividing the normalized activity time span. Thus the FS analysis in each data set could be described as follows. For the user $u$, a new rating series $R S_{u}^{T}$ consists of the sum of the ratings in each time interval. If we denote the sum of the ratings in time interval $\left[t-\frac{1}{T}, t\right)\left(t=\frac{1}{T}, \frac{2}{T}, \ldots, 1\right.$, 

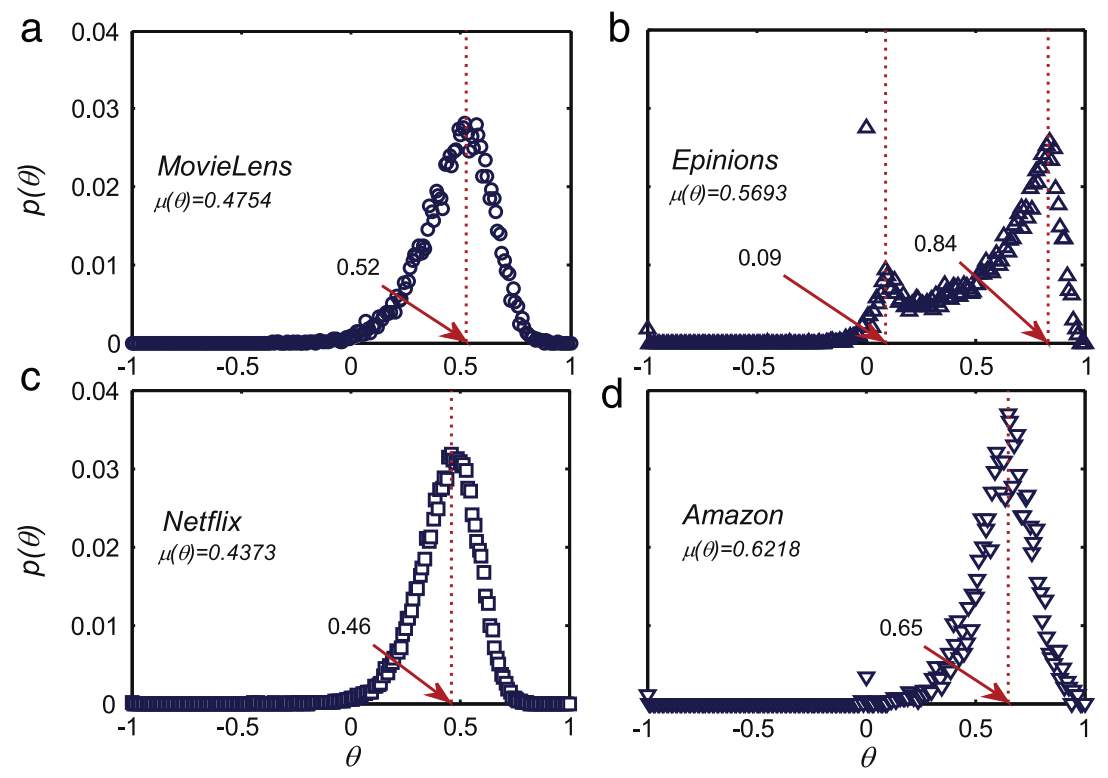

Fig. 10. The probability distribution $p(\theta)$ of rating similarity $\theta$ between the user rating and the object quality. The object quality is quantified by averaging all of the ratings delivered to the object. Thus, for each user the rating series and the object quality series consist of two vectors. The Pearson correlation coefficient between the two vectors is denoted as the rating similarity $\theta$. Panels (a)-(d) show the distribution $p(\theta)$ of the rating similarity $\theta$ for different data sets. The mean values of the rating similarity $\theta$ in the MovieLens, Epinions, Netflix, and Amazon data sets are $0.4754,0.4373,0.5693$ and 0.6218 respectively, which implies the positive correlations between the ratings and the global qualities in the aggregate level, and for the Amazon data set the users' ratings are more dependent with the object qualities than any others. It also can be seen that, in the MovieLens, Epinions, Netflix, and Amazon data sets, the modes of the values of the rating similarity $\theta$ is $0.52,0.84,0.46$ and 0.65 respectively.

and it is $\left[\frac{T-1}{T}, 1\right]$ for the last interval) as

$$
f_{u}(t)=\sum_{i=1}^{N_{u}(t)} r_{i}
$$

where $N_{u}(t)$ is the number of ratings user $u$ delivered during the time interval, then the rating series $R S_{u}^{T}=\left\{f_{u}\left(\frac{1}{T}\right), f_{u}\left(\frac{2}{T}\right), \ldots\right.$, $\left.f_{u}(1)\right\}$. Therefore, the temporal average value $\mu_{u}$ can be read as

$$
\mu_{u}=\frac{1}{T} \sum_{t=\frac{1}{T}}^{1} f_{u}(t)
$$

and the standard deviation $\sigma_{u}$ is quantified as

$$
\sigma_{u}=\left(\frac{1}{T-1} \sum_{t=\frac{1}{T}}^{1}\left[f_{u}(t)-\mu_{u}\right]^{2}\right)^{\frac{1}{2}} .
$$

Then, we could obtain the value of FS exponent $\beta$ by linearly fitting the scatters $(\log \mu, \log \sigma)$ of all users.

In the null model, users are set to uniformly and randomly deliver integral ratings from the rating range $\left[r_{\min }, r_{\max }\right]$, where the $r_{\min }$ and $r_{\max }$ respectively denote the minimum and maximum scores that each user can rate. Therefore, the empirical correlation of the user rating behaviors is removed, so that the FS analysis could solely reflect the correlation embedded in the structure of the data sets. The results of the FS analysis for null model are shown in Fig. 11, from which, one could find that the fluctuation scaling exponent $\beta$ equals $0.8534,0.9135,1.0731$ and 0.7619 for the MovieLens, Epinions, Netflix and Amazon data sets respectively. Therefore, the results display the evidence of the correlation of online user collective behaviors in the data sets, which may be caused by the distribution of inter-event times of the user actions or the inevitable interactions among users within these web-based systems etc.

Therefore, the above results reveal the non-trivial correlation between the user ratings and the object qualities. However, comparing with the rating behaviors of the Amazon data set, the lower mean value of the rating similarity $\theta$ for other systems report that, ratings for movies/reviews are more independent with the object qualities than those ratings for books. Besides, we also find that for the Amazon and Epinions data sets, a few users tend to give ratings that are irrelevant or even opposite to the object qualities. 

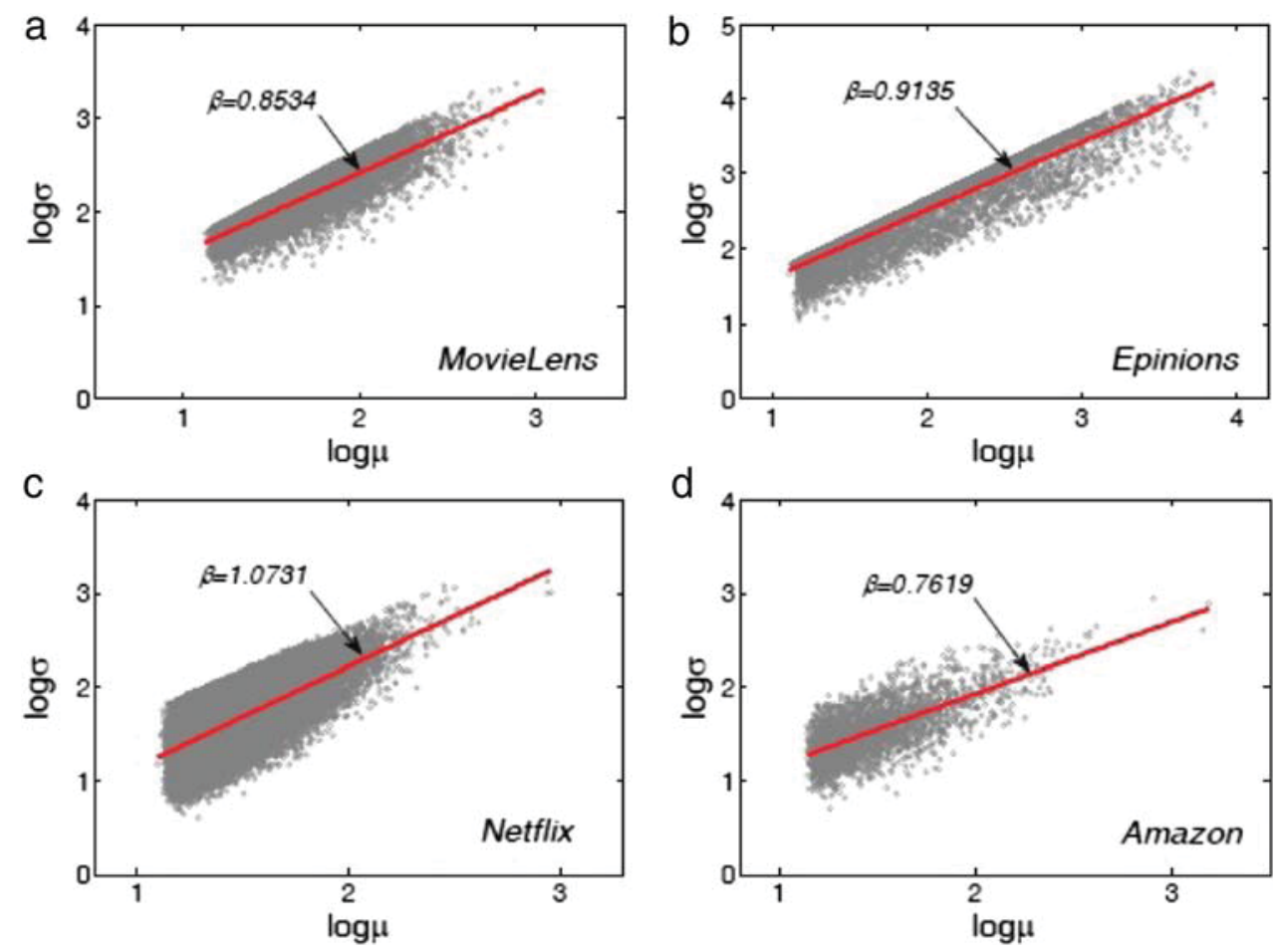

Fig. 11. Fluctuation scaling plots for the four data sets. In the fluctuation scaling (FS) analysis, the temporal average value $\mu_{u}$ and the standard deviation $\sigma_{u}$ of the rating sequence of a user, say $u$, are related by the correlation $\sigma_{u} \sim \mu_{u}^{\beta}$. The value of the fluctuation scaling exponent $\beta$ is given by the least square fitting for the points $(\log \mu, \log \sigma)$ for all the users, which lies in range [1/2,1], where $\beta=1 / 2$ means the independence of the users' behaviors while $\beta=1$ implies that the users behaviors are fully correlated. In null model, the users are set to randomly deliver the ratings, while the timestamps still keep the same as the empirical ones. Then, the FS analysis of the null model could solely reflect the correlation of the user rating behaviors embedded in the structure of the data sets. The panels (a)-(d) show that the FS exponent $\beta$ equals $0.8534,0.9135,1.0731$ and 0.7619 respectively for the MovieLens, Epinions, Netflix and Amazon data sets. Therefore, the correlation of the user rating behaviors is relatively evident.

\section{Correlation dynamics for user rating behaviors}

Furthermore, we investigate the rating similarity $\theta$ to uncover the differences of the correlation evolution patterns for different users. First of all, users are collected into six groups according to their degree $k$, i.e., the number of ratings they delivered in the whole data set, since the special roles of the degree in online user activity patterns [38-40]. In each data set, the users are grouped by the degree ranging in $[k(g), k(g) \times d]$ for the first five groups, where $k(g)$ denotes the minimum degree in group $g$ and the common ratio $d=[k(g+1)-1] / k(g)$. As we described in the section of Data description, the minimum degree in each data set is $k_{\min }=k(1)=100$. The common ratio $d$ is $1.53,2.09,1.50$, and 1.58 for the MovieLens, Epinions, Netflix, and Amazon data sets respectively. Then, we could trace the reactions of different users to the displayed quality of an object before s/he rates on it. Here, the displayed quality of an object is a function of the timestamp of the user rating. If a user, say $u$, rate object $o$ at time $t$, the displayed quality of object $o$ can be computed by averaging all its ratings given by users just before time $t$. Hence, the dynamical correlation between the ratings and the displayed qualities can be measured as follows.

Suppose the user $u$ belongs to group $g$ ( $g=1,2, \ldots, 6$ ) and had rated $m_{u}$ ratings during the activity span, we firstly focus on the correlation dynamics in given time interval $\left[t-\frac{1}{T}, t\right)$, in which $t=\frac{1}{T}, \frac{2}{T}, \ldots, \frac{T-1}{T}, 1$ (It is $\left[\frac{T-1}{T}, 1\right]$ for the last time interval). The number of ratings user $u$ delivered during this period are denoted as $m_{u}^{\prime}(t)$. For each rating $r_{i}$, there is a displayed quality $q_{r_{i}}$ corresponding to the timestamp of the rating record. Thus, the vectors consisted of the ratings and the displayed qualities can be expressed as $R_{u}(t)=\left\{r_{1}, r_{2}, \ldots, r_{m_{u}^{\prime}(t)}\right\}$ and $Q_{u}(t)=\left\{q_{r_{1}}, q_{r_{2}}, \ldots, q_{r_{m_{u}^{\prime}}(t)}\right\}$, respectively. Then the dynamical correlation $\rho_{u}(t)$ of user $u$ at time $t$ is defined as,

$$
\rho_{u}(t)=\frac{1}{m_{u}^{\prime}(t)} \sum_{i=1}^{m_{u}^{\prime}(t)} \frac{\left[r_{i}-\bar{r}_{u}(t)\right]\left[q_{r_{i}}-\bar{q}_{u}(t)\right]}{\sigma_{r_{u}}(t) \sigma_{q_{u}}(t)},
$$

where $\bar{r}_{u}(t)=\frac{1}{m_{u}^{\prime}(t)} \sum_{i=1}^{m_{u}^{\prime}(t)} r_{i}, \bar{q}_{u}(t)=\frac{1}{m_{u}^{\prime}(t)} \sum_{i=1}^{m_{u}^{\prime}(t)} q_{r_{i}}, \sigma_{r_{u}}(t)=\sqrt{\frac{1}{m_{u}^{\prime}(t)} \sum_{i=1}^{m_{u}^{\prime}(t)}\left(r_{i}-\bar{r}_{u}(t)\right)^{2}}$ and $\sigma_{q_{u}}(t)=$ $\sqrt{\frac{1}{m_{u}^{\prime}(t)} \sum_{i=1}^{m_{u}^{\prime}(t)}\left(q_{r_{i}}-\bar{q}_{u}(t)\right)^{2}}$. Consequently, the average dynamical correlation $\langle\rho(t)\rangle_{g}$ for users in group $g$ at time $t$ can 

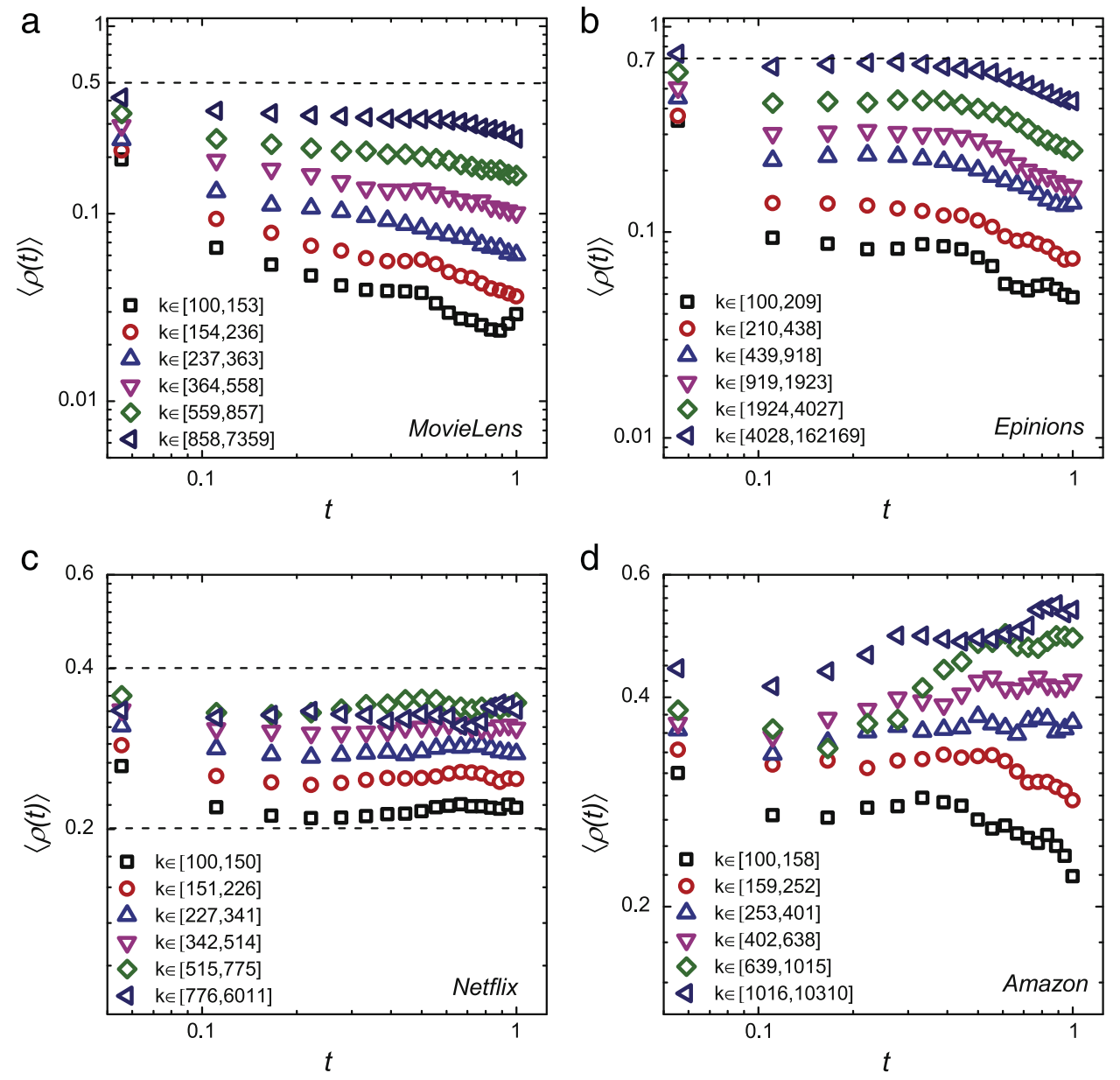

口 Group $1 \bigcirc$ Group $2 \Delta$ Group $3 \nabla$ Group $4 \diamond$ Group $5 \triangleleft$ Group 6

Fig. 12. (Color online) The evolution of the dynamical correlation $\langle\rho(t)\rangle$ for different users. The user degree could be a key element in the user activity patterns according to previous literatures $[38,39]$. Therefore, for each data set the users are divided into six groups in terms of their degree, generated by the number of objects each of them rated. The dynamical correlation $\langle\rho(t)\rangle$ is measured by the $P$ between the user rating series and the displayed quality series corresponding to time $t$ (see Section Correlation dynamics for user rating behaviors for related details and definitions). The panels (a)-(d) show the evolution of the dynamical correlation $\langle\rho(t)\rangle$ for different users, where from Group 1 to Group 6 each group contains the users with the degrees from the least to the largest respectively. One could find that, in the MovieLens, Epinions, and Netflix data sets, the dynamical correlation $\langle\rho(t)\rangle$ decreases versus time $t$ and presents specific evolution pattern for all users. Whereas, in the Amazon data set it exhibits inconsistent evolution trends for different users as the time increases.

be read as,

$$
\langle\rho(t)\rangle_{g}=\frac{1}{N_{g}} \sum_{u \in \Omega_{g}} \rho_{u}(t)
$$

where $N_{g}$ is the number of the users in group $g$ and $\Omega_{g}$ is the set that contains all the users in group $g$. The higher value of the average dynamical correlation $\left\langle\rho\left(t_{s}\right)\right\rangle_{g}$ is, the higher possibility users in Group $g$ rate objects depending on the displayed qualities.

The evolution trends of the average dynamical correlation $\langle\rho(t)\rangle$ are shown in Fig. 12. One could find, the larger the user degree is, the higher the average dynamical correlation $\langle\rho(t)\rangle$ will be, which suggests that the users who delivered more ratings are more likely to give ratings approximated to the object quality they have seen. Meanwhile, the evolution processes of the average dynamical correlation $\langle\rho(t)\rangle$ for the MovieLens, Epinions, and Netflix data sets are remarkably different with the ones observed in the Amazon data set. For the MovieLens, Epinions, and Netflix data sets, as the time $t$ increases, the evolution trends of the average dynamical correlation $\langle\rho(t)\rangle$ are similar for different groups. Specifically, for the MovieLens data set, the average dynamical correlation $\langle\rho(t)\rangle$ monotonously decreases for all users. For the Epinions data set, at the start of the user online activities the average dynamical correlation $\langle\rho(t)\rangle$ decreases, and during the period from $t=0.11$ to $t=0.33$ little change can be found, and during the rest of the activity span it would decreases again. For the Netflix data set, the average dynamical correlation $\langle\rho(t)\rangle$ tends to decline at the first, and then slightly fluctuates around a small value ranging in 

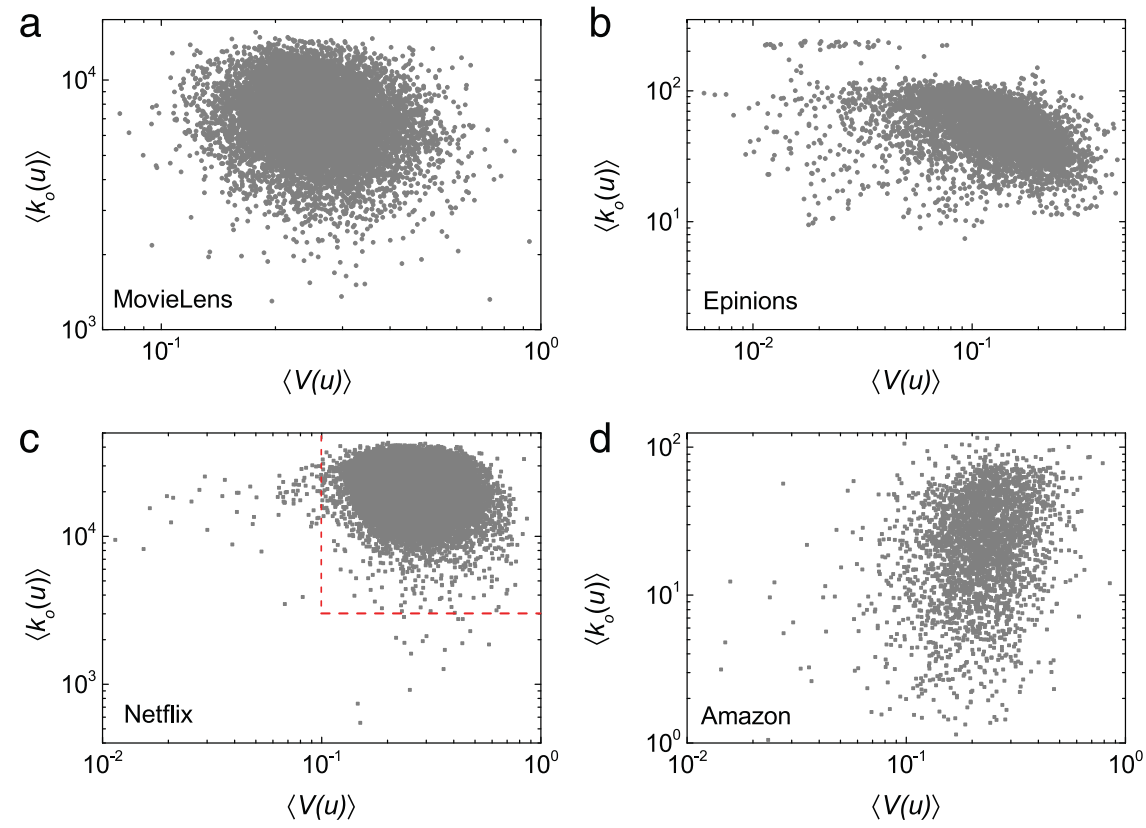

Fig. 13. The scatter plot of the overall variation coefficient $\langle V(u)\rangle$ versus the average object degree $\left\langle k_{o}(u)\right\rangle$. Panels (a)-(d) show the respective results for the MovieLens, Epinions, Netflix and Amazon data sets. The coordinate of each point in the panels consists of the overall variation coefficient $\langle V(u)\rangle$, defined as the ratio of the standard deviation to the mean of the user's whole rating sequence, and the average object degree $\left\langle k_{0}(u)\right\rangle$, calculated by averaging the degree over all of the objects that the user $u$ rated. As one could find, the point $\left(\langle V(u)\rangle,\left\langle k_{0}(u)\right\rangle\right)$ locates in each panel without any obvious regularity, suggesting the non-significant correlation between the overall variation coefficient $\langle V(u)\rangle$ and the average object degree $\left\langle k_{o}(u)\right\rangle$.

$[0.2,0.4]$. The above results indicate that, when users evaluate movies or reviews, they are likely to have a general reaction pattern to the object, which could be reflected by the increasingly insignificant but special correlation patterns between the ratings and the displayed qualities. The patterns are reasonable to some extent. As a user just enter an online system, his/her attitudes could be largely impacted by the others' ratings, resulting in the closely correlation between the ratings and the displayed qualities. With the rating experience enriched, the user are more likely to give ratings relying on personal preference. Therefore, it could be collectively seen that the correlation declines. However, for the Amazon data set, the average dynamical correlation $\langle\rho(t)\rangle$ presents no general pattern for all the users, which implies that some users rate the books increasingly close to the displayed quality while for others the deviation of ratings from the displayed qualities is growing.

Generally speaking, the user rating behaviors are influenced by the quality of the evaluation object. However, the influence would be increasingly insignificant as the time increases. Besides, different data sets are characterized by different evolution processes of the correlation between the ratings and the displayed qualities.

\section{Correlation between the user preference diversity and the object degree}

The object degree is another important factor that may influence the user rating behaviors $[26,40]$. To collectively clarify whether the user rating preference diversity is related to the object degree, we investigate the correlation between the user preference diversity and the object degree. In this section, for a user, says $u$, we define the overall variation coefficient $\langle V(u)\rangle$ as the variation coefficient over all the ratings s/he delivered which can be read as,

$$
\langle V(u)\rangle=\frac{\sigma(u)}{\mu(u)},
$$

where the $\mu(u)=\mu_{u}\left(t_{\max }\right)$ and $\sigma(u)=\sigma_{u}\left(t_{\max }\right)$ (as aforementioned, $t_{\max }=1$ ). Correspondingly, we average the object degree over all the objects rated by user $u$, denoted as $\left\langle k_{0}(u)\right\rangle$, which can be read as,

$$
\left\langle k_{o}(u)\right\rangle=\frac{\sum_{o \in \Omega_{u}} k(o)}{N_{o}(u)},
$$

where $\Omega_{u}$ is a set containing all the objects that user $u$ rated, $k(o)$ is the degree of object $o$ and $N_{o}(u)$ denotes the corresponding number of the objects.

The results of the overall variation coefficient $\langle V(u)\rangle$ versus the average object degree $\left\langle k_{0}(u)\right\rangle$ are shown in Fig. 13. One can find that, a specific value of the overall variation coefficient $\langle V(u)\rangle$ could correspond to almost all scope of the value of the average object degree $\left\langle k_{o}(u)\right\rangle$. For example, in the Netflix data set, most values of the overall variation coefficient $\langle V(u)\rangle$ 
locates in $[0.1,1]$ as well as each of the value can be found to pair with various average object degree $\left\langle k_{0}(u)\right\rangle$ ranging in $\left[3 \times 10^{3}, 4 \times 10^{4}\right]$, suggesting that the user preference diversity has non-obvious correlation with the object degree. That is to say, whether the user prefers to deliver high/low ratings or to give more diverse ratings is unlikely to rely on the popularity of an object (based on the simple and well-established assumption-the larger the object degree is, the more popular the object among users). The users whose rating preference diversities are small may give scores on the objects with various object degrees, so do the users whose rating preference diversities are large. Therefore, the rating preference could be an internal properties embedded in the online user behavior regardless of the object degree, which needs additional efforts to gain more specified results.

\section{Conclusion and discussions}

In this paper, we empirically investigated the evolution properties of the online user preference diversity, which is measured by the average variation coefficient $\langle V(t)\rangle$. The empirical results indicate that, for movies and reviews, the user preferences become increasingly diverse at the early stage of their life span, and then become more and more centralized. We proposed a Markov model to regenerate the collective properties of online user behaviors. In this model, the transition probability between user's two consecutive behaviors is generated from a Gaussian distribution with the empirical standard deviation. The results suggest that with the proper value of parameter $\alpha$, the characteristics of the user behaviors can be well modeled by the Markov process for some online systems like MovieLens and Epinions. However, for others like Netflix and Amazon, the Markov process could not regenerate the statistical property of the user behaviors. Therefore, when exploring the behavior patterns with the anchoring bias or the memory effect, it is necessary to inspect whether the online user rating behaviors could be reproduced by the Markov process. Besides, a detailed analysis of the correlation between the user rating and the object quality suggests that, the user rating are increasingly uncorrelated with the object quality and the correlation $\theta$ for different users exhibit consistent evolution trend, which would provide new sights about the users' reactions to the object qualities from the evolution point of view. Additionally, the user rating preference diversity and the object degree present non-obvious correlations.

Online user preference could be affected by the social influence $[8,41,42]$ and the recommendation systems [43-45]. Recent advances suggest that, the human social behaviors present remarkable distinct properties against the differences in age, sex and personality etc., both on the collective and individual levels [42,46-48]. Consequently, how to identify the evolution characteristics of online user preference with these attributes are still open questions, which requires not only the details of the user profiles but also the trade-off between the sample size and the statistical methods. It is also very important to dig out the necessary condition when the Markov model could be used to model the online collective behaviors. Even though there are still these unsolved problems, our findings are very helpful for deeply understanding the evolution properties of the online collective behaviors and precisely modeling the complex online collective behaviors.

\section{Acknowledgments}

This work was supported by the National Natural Science Foundation of China (Nos. 71371125, 61374177, 71271126, 71271036), the Program for Professor of Special Appointment (Eastern Scholar) at Shanghai Institutions of Higher Learning, the Shuguang Program Project of Shanghai Educational Committee (Grant No. 14SG42). JGL was supported by the Sino-Swiss Science and Technology Cooperation (No. EG 09-032016).

\section{References}

[1] D.J. Watts, A twenty-first century science, Nature 445 (2007) 489-489.

[2] R.D. Malmgren, D.B. Stouffer, A.S. Campanharo, L.A.N. Amaral, On universality in human correspondence activity, Science 325 (2009) $1696-1700$.

[3] A. Vespignani, Predicting the behavior of techno-social systems, Science 325 (2009) 425-428.

[4] J.L. Iribarren, E. Moro, Impact of human activity patterns on the dynamics of information diffusion, Phys. Rev. Lett. 103 (2009) 038702.

[5] N. Christakis, J. Fowler, The spread of obesity in a large social network over 32 years, N. Engl. J. Med. 357 (2007) 370-379.

[6] J.G. Liu, Z.L. Hu, Q. Guo, Effect of the social influence on topological properties of user-object bipartite networks, Eur. Phys. J. B 86 (2013) 1-11.

[7] J. Fowler, N. Christakis, Estimating peer effects on health in social networks: A response to Cohen-Cole and Fletcher; and Trogdon, Nonnemaker, Pais, J. Health Econ. 27 (2008) 1400-1405.

[8] J.P. Onnela, F. Reed-Tsochas, Spontaneous emergence of social influence in online systems, Proc. Natl. Acad. Sci. USA 107 (2010) $18375-18380$.

[9] A.L. Barabási, The origin of bursts and heavy tails in human dynamics, Nature 435 (2005) 207-211.

[10] C. Castellano, S. Fortunato, V. Loreto, Statistical physics of social dynamics, Rev. Modern Phys. 81 (2009) 591-646.

[11] J.G. Oliveira, A.L. Barabási, Darwin and Einstein correspondence patterns, Nature 437 (2005) 1251-1251.

[12] D. Lazer, A.S. Pentland, L. Adamic, S. Aral, A.L. Barabási, D. Brewer, et al., Life in the network: The coming age of computational social science, Science 323 (2009) 721-723.

[13] X.P. Han, T. Zhou, B.H. Wang, Modeling human dynamics with adaptive interest, New J. Phys. 10 (2008) 073010.

[14] T. Zhou, Z. Kuscsik, J.G. Liu, M. Medo, J.R. Wakeling, Y.C. Zhang, Solving the apparent diversity-accuracy dilemma of recommender systems, Proc. Natl. Acad. Sci. USA 107 (2010) 4511-4515.

[15] Z.K. Zhang, C. Liu, A hypergraph model of social tagging networks, J. Stat. Mech. 10 (2010) P10005.

[16] C.X. Zhang, Z.K. Zhang, C. Liu, An evolving model of online bipartite networks, Physica A 392 (2013) 6100-6106.

[17] A.M. Kaplan, M. Haenlein, Users of the world, unite! The chanllenges and opportunities of social media, Bus. Horiz. 53 (2010) $59-68$.
[18] J.H. Kietzmann, K. Hermkens, I.P. McCarthy, B.S. Silvestre, Social Media? Getserious! Understanding the functional building blocks of social media, Bus. Horiz. 54 (2011) 241-251.

[19] Z. Dezsö, E. Almaas, A. Lukács, B. Rácz, I. Szakadát, A.L. Barabási, Dynamics of information access on the web, Phys. Rev. E 73 (2006) 066132. 
[20] A.L. Barabási, Network science: Luck or reason, Nature 489 (2012) 507-508

21] M. Schich, C. Song, Y.Y. Ahn, A. Mirsky, M. Martino, A.L. Barabási, D. Helbing, A network framework of cultural history, Science 345 (2014) $558-562$.

22] D. Rybski, S.V Buldyrev, S. Havlin, F. Liljeros, H. A. Makse, Scaling laws of human interaction activity, Proc Natl. Acad. Sci. USA 106 (2009) 12640 - 12645.

[23] D. Rybski, S.V. Buldyrev, S. Havlin, F. Liljeros, H.A. Makse, Communication activity in a social network: Relation between long-term correlations and inter-event clustering, Sci. Rep. 2 (2012) 560.

24] Z.M. Yang, Z.K. Zhang, T. Zhou, Anchoring bias in online voting, Europhys. Lett. 100 (2012) 68002.

25] L. Hou, X. Pan, Q. Guo, J.G. Liu, Memory effect of the online user preference, Sci. Rep. 4 (2014) 6560.

[26] Y.L. Zhang, Q. Guo, J. Ni, J.G. Liu, Memory effect of the online rating for movies, Physica A 417 (2015) 261-266.

27] M. Karsai, K. Kaski, A. Barabási, J. Kertész, Universal features of correlated bursty behaviour, Sci. Rep. 2 (2012) 397.

28] Z.D. Zhao, Z.K. Zhang, T. Zhou, Z.G. Huang, Y.C. Lai, Emergence of scaling in human-interest dynamics, Sci. Rep. 3 (2013) 3472.

29] S. Brin, L. Page, Reprint of: The anatomy of a large-scale hypertextual web search engine, Comput. Netw. 56 (2012) 3825-3833.

[30] N. Craswell, M. Szummer, Random walks on the click graph, in: SIGIR'07: Proc. 30th Annual Int. ACM SIGIR CRDIR, ACM, Amsterdam, The Netherlands. New York, NY, USA, 2007, pp. 239-246.

[31] R. Fagin, A.R. Karlin, J. Kleinberg, P. Raghavan, S. Rajagopalan, R. Rubinfeld, et al., Random walks with "back buttons”, Ann. Appl. Probab. 11 (2011) $810-862$.

[32] M.R. Meiss, B. Gonçalves, J.J. Ramasco, A. Flammini, F. Menczer, Agents, bookmarks and clicks: A topical model of web navigation, in: Proceedings of the 21st ACM Conference on Hypertext and Hypermedia, ACM, 2010, pp. 229-234.

[33] F. Chierichetti, R. Kumar, P. Raghavan, T. Sarlós, Are web users really Markovian? in: Proceedings of the 21st International Conference on World Wide Web, ACM, 2012, pp. 609-618.

[34] Z. Eisler, I. Bartos, J. Kertesz, Fluctuation scaling in complex systems: Taylor's law and beyond, Adv. Phys. 57 (2008) 89-142.

[35] A. van de Rijt, S.M. Kang, M. Restivo, A. Patil, Field experiments of success-breeds-success dynamics, Proc. Natl. Acad. Sci. USA 111 (2014) 6934-6939,

[36] P. Massa, P. Avesani, Trust-aware bootstrapping of recommender systems, in: Proceedings of ECAI 2006 Workshop on Recommender Systems, 2006, pp. 29-33.

[37] K.I. Goh, A.L. Barabási, Burstiness and memory in complex systems, Europhys. Lett. 81 (2008) 48002.

[38] Q. Guo, F. Shao, Z.L. Hu, J.G. Liu, Statistical properties of the personal social network in the Facebook, Europhys. Lett. 104 (2013) 28004.

[39] J.G. Liu, Z.M. Ren, Q. Guo, B.H. Wang, Node importance ranking of complex networks, Acta Phys. Sinica 62 (2013) 178901

[40] Q. Guo, W.J. Song, J.G. Liu, Ultra-accurate collaborative information filtering via directed user similarity, Europhys. Lett. 107 (2014) 18001.

[41] W.A. Mason, F.R. Conrey, E.R. Smith, Situating social influence processes: Dynamic, multidirectional flows of influence within social networks, Pers. Soc. Psychol. Rev. 11 (2007) 279-300.

[42] K. Lewis, M. Gonzalez, J. Kaufman, Social selection and peer influence in an online social network, Proc. Natl. Acad. Sci. USA 109 (2012) 68-72.

[43] G. Adomavicius, A. Tuzhilin, Toward the next generation of recommender systems: A survey of the state-of-the-art and possible extensions, IEEE Trans. Knowl. Data Eng. 17 (2005) 734-749.

[44] U. Hanani, B. Shapira, P. Shoval, Information filtering: Overview of issues, research and systems, User Model. User-Adapt. Interact. 11 (2001) 203-259.

[45] P. Resnick, H.R. Varian, Recommender systems, Commun. ACM 40 (1997) 56-58.

[46] J. Saramäki, E.A. Leicht, E. López, S.G.B. Roberts, F. Reed-Tsochas, R.I.M. Dunbar, Persistence of social signatures in human communication, Proc. Natl. Acad. Sci. USA 111 (2014) 942-947.

[47] H.H. Jo, J. Saramäki, R.I.M. Dunbar, K. Kaski, Spatial patterns of close relationships across the lifespan, Sci. Rep. 4(2014) 6988.

[48] V. Palchykov, K. Kaski, J. Kertész, A.L. Barabási, R.I.M. Dunbar, Sex differences in intimate relationships, Sci. Rep. 2 (2012) 370. 